\title{
LONG GRB WITH ADDITIONAL HIGH ENERGY MAXIMA AFTER THE END OF THE LOW ENERGY T $_{90}$ INTERVALS
}

\author{
ARKHANGELSKAJA IRENE \\ Department of Experimental Nuclear Physics and Cosmophysics \\ National Research Nuclear University "MEPhI" \\ Kashirskoe shosse, 31, Moscow,115409, Russia \\ irene.belousova@usa.net \\ ZENIN ALEXANDER, KIRIN DMITRY and VOEVODINA ELENA \\ National Research Nuclear University "MEPhI" \\ Kashirskoe shosse, 31, Moscow,115409, Russia
}

\begin{abstract}
Now GRB high energy $\gamma$-emission was observed mostly by detectors onboard Fermi and Agile satellites. During most part of GRB high energy $\gamma$-emission registered some later than low energy trigger and lasts several hundreds of seconds, but its maxima are within low energy t90 intervals both for short and long bursts. But GRB090323, GRB090328 and GRB090626 temporal profiles have additional maxima after low energy t90 intervals finished. These bursts temporal profile analysis have shown that faint peaks in low energy bands close to the ends of low energy t ${ }_{90}$ intervals preceded such maxima. Moreover, these events low energy spectral index $\beta$ behavior differs from usual GRB one according to preliminary analysis. We suppose that these GRB could be separated as different GRB type. In presented article this new GRB type properties are discussed.
\end{abstract}

Keywords: GRB; high energy gamma-emission.

\section{Introduction}

There were several periods of GRB observation in high-energy region. Firstly GRBs high energy component was observed by COMPTEL and EGRET onboard CGRO in 1991 (see Ref. 1). The widest energy range for GRBs $\gamma$-emission registration by CGRO was $10 \mathrm{keV}-20 \mathrm{GeV}$. Some tens of GRBs were detected simultaneously by all 4 experiments onboard CGRO: BATSE, OSSE, COMPTEL, and EGRET ${ }^{2}$. But during GRB 970417A photons with energies above $650 \mathrm{GeV}$ were registered according to preliminary data analysis from the Milagrito - see Ref. 3.

With beginning functionality of Russian satellite CORONAS-F (NORAD cata$\log$ number 26873, ID 2001-032A operation period from July 31, 2001 to December 6,2005 ) one of the next steps of GRBs high energy emission investigation by satellite data was started. AVS-F apparatus onboard CORONAS-F allows to detect GRBs in energy bands $0: 1-22 \mathrm{MeV}$ and $2-260 \mathrm{MeV}$ by data of last flight calibrations ${ }^{4,5}$. 

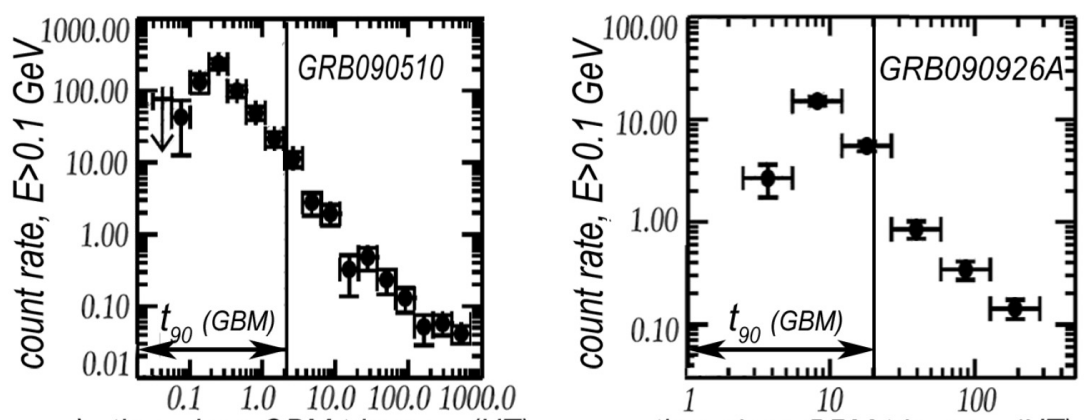

a) time since GBM trigger, s (UT)

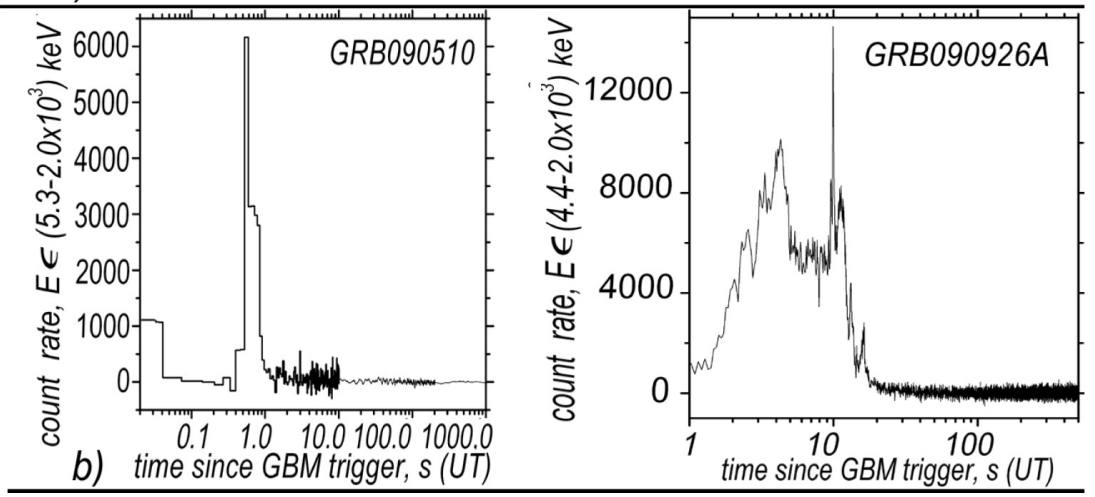

Fig. 1. The temporal profiles of GRB 090510 and GRB 090926A by: (a) Fermi/LAT data (adopted from Ref. 11) and (b) Fermi/GBM data. The arrows have shown t90 on GBM data.

In the present time high energy emission from GRBs could be observed by detectors onboard satellites AGILE and Fermi operated since April 2007 (Ref. 6) and June 2008 (Ref. 7) correspondingly. The Gamma-Ray Imaging Detector (GRID) onboard AGILE covers one fifth of the sky in the $30 \mathrm{MeV}-30 \mathrm{GeV}$ energy range Ref. 8. The Large Area Telescope (LAT) onboard Fermi provides energy coverage from $20 \mathrm{MeV}$ to $300 \mathrm{GeV}$, the temporal structure registration for even the shortest GRBs and localization of GRBs with high precision sufficiently for follow-up observations by other experiments ${ }^{7,9}$. Fermi gamma-ray observatory registered approximately 850 identified GRBs since 2008 July using GBM and 27 GRBs using LAT. The highest energy of photons was registered during GRB 090902B and it was $\sim 33 \mathrm{GeV}$ (Ref. 7). Several tens of bursts were registered by AGILE but only several percents of these events had high energy $\gamma$-component ${ }^{10}$.

Extended high energy $\gamma$-emission up to some hundreds of seconds was registered during several bursts by Fermi/LAT as it was mentioned in the Ref. 11. Such high energy gamma component observed both during long (in particular, GRB 090926) and short (for example, GRB 090510) events. The examples of GRBs temporal profiles with extended high energy emission registered by Fermi are presented at the figure 1. Also several GRBs with extended high energy $\gamma$-emission were observed by 
AGILE, for example GRB080514B was observed in the energy band $30 \mathrm{MeV}-30 \mathrm{GeV}$ (see Ref. 12). During most part of long GRB high energy $\gamma$-emission registered some later than low energy trigger some tens times longer than duration of bursts ${ }^{11,13}$, but in several cases one observed before GRB and could be interpreted as high energy precursors $^{14}$. But several events with high energy emission duration less then low one or similar temporal profiles properties in GBM and LAT energy bands were observed (for example, GRB 090217A and GRB 110721A correspondingly — see Refs. 15, 16).

\section{The New Type of Long GRB with High Energy $\gamma$-Emission}

As it was mentioned in the previous section, usually high energy $\gamma$-emission during long GRB registered some later than low energy trigger and lasts several hundreds of seconds, but its maxima are within low energy t90 intervals. Such events extended component duration was some tens times longer than burst duration ${ }^{11,13,14}$. The GRBs duration and peak positions in the low and high energy band are presented in the Table 1. According to preliminary data analysis there was no correlation between GRB duration $t_{90}$ in the low energy band and lasting of high energy gamma component — see Table 1.

However, in contradiction to other GRB, there were additional maxima on the temporal profiles of GRB090323 and GRB090328 in high energy band located outside low energy t go $_{9}$ intervals - see Fig. 2 in comparison with Fig. 1 and Ref. 17. Faint peaks in the low energy band located close to the end of $t_{90}$ intervals according to $\mathrm{NaI}(\mathrm{Tl})$ detectors data preceded high energy maxima for such bursts. GRB090626

Table 1. The characteristics of GRBs temporal profiles.

\begin{tabular}{|c|c|c|c|c|}
\hline GRB & $\begin{array}{l}\text { Low } \\
\text { energy } \\
\text { t90, s }\end{array}$ & $\begin{array}{l}\text { Approximately } \\
\text { high energy } \\
\text { duration }^{\mathrm{a}}\end{array}$ & $\begin{array}{l}\text { Low energy } \\
\text { approximately } \\
\text { peak position }\end{array}$ & $\begin{array}{l}\text { High energy approximately } \\
\text { peak position since } \\
\text { GBM burst begin, } \mathrm{s}^{\mathrm{a}}\end{array}$ \\
\hline 100116A & & $1.5 \times 10^{2}$ & & 90 \\
\hline 091003 & 21,1 & $2 \times 10^{3}$ & $\begin{array}{l}0.5 ; 2.5 ; 5 ; 10 \\
15.5 ; 19\end{array}$ & $<2$ \\
\hline 090926A & 20 & $2 \times 10^{3}$ & $4 ; 10$ & 8 \\
\hline 090902B & 21 & $1.8 \times 10^{2}$ & $10 ; 14 ; 19$ & 8 \\
\hline 090626 & $\sim 70$ & $4 \times 10^{2}$ & $\begin{array}{l}0.6 ; 1.6 ; 3.6 ; 17 \\
19 ; 22 ; 34 ; 45\end{array}$ & 110 \\
\hline 090510 & 0,3 & $2 \times 10^{3}$ & $\begin{array}{l}0.1 ; 0.55 ; 0.65 \\
0.75 ; 0.85\end{array}$ & 0.7 \\
\hline 090328 & 80 & $6 \times 10^{3}$ & $5 ; 13 ; 50$ & 300 \\
\hline 090323 & 150 & $3 \times 10^{3}$ & $12 ; 60 ; 130$ & 200 \\
\hline 090217 & 32,8 & 30 & $\begin{array}{l}6 ; 10 ; 19 ; \\
29 ; 38\end{array}$ & $<2$ \\
\hline 081024B & .8 & 10 & $0.2 ; 0.5$ & $<0.2$ \\
\hline 080916C & 66 & $1 \times 10^{3}$ & $3 ; 18 ; 42$ & 6 \\
\hline $080825 \mathrm{C}$ & & $4 \times 10^{2}$ & $4 ; 10 ; 12 ; 15$ & 2.5 \\
\hline
\end{tabular}

a According to results of preliminary data analysis. 

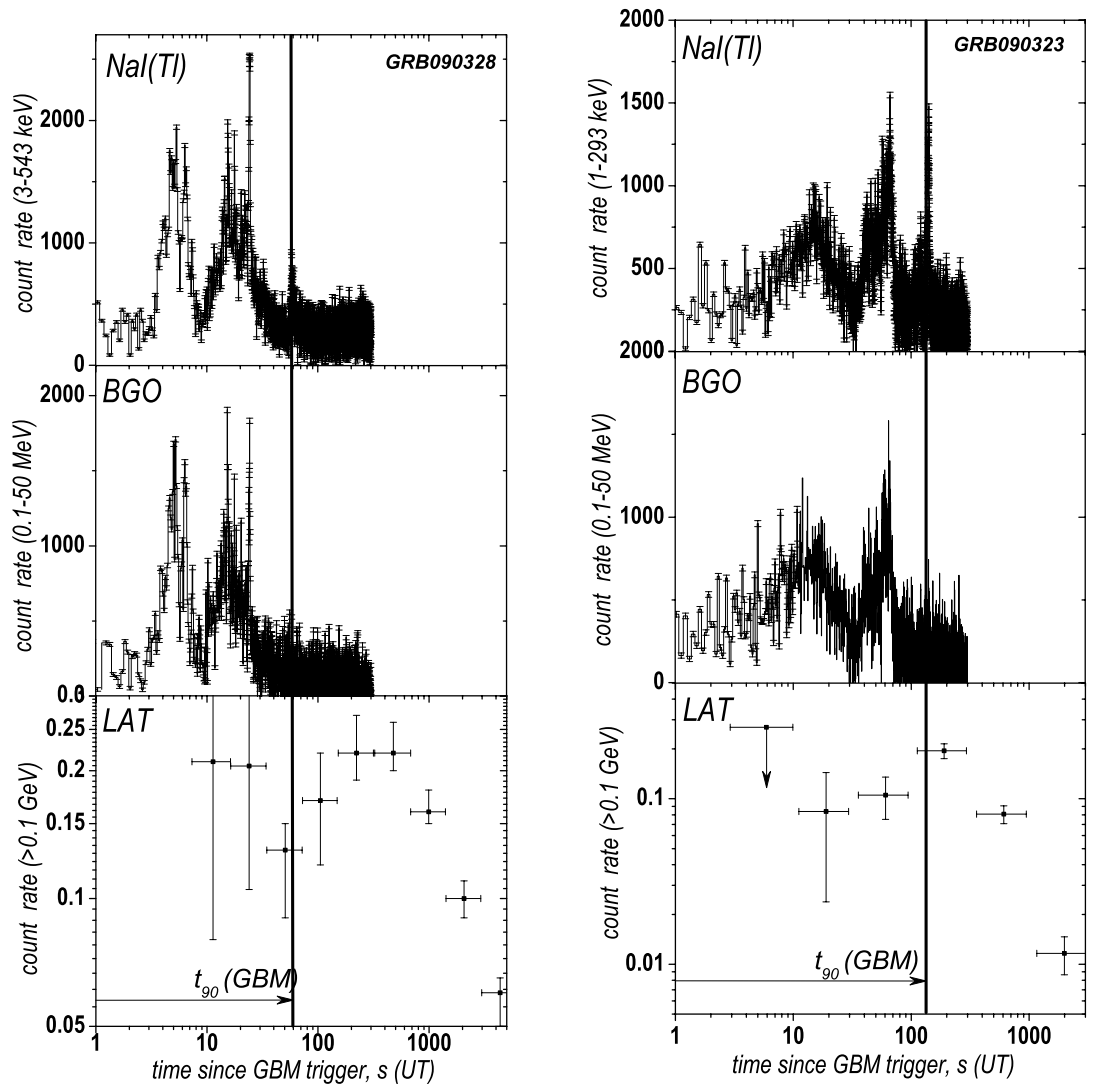

Fig. 2. The temporal profiles of GRB090328 and GRB090323 by Fermi/LAT data (adopted from Ref. 11) and Fermi/GBM data. The arrows have shown t90 interval on GBM data.

has the similar temporal profiles characteristics ${ }^{17}$. According to the temporal profile analysis preliminary results, the whole duration of the high energy $\gamma$-component and its peak position during such events are not depend on characteristics of temporal profiles in low energy band. We suppose that these bursts could be separated as different GRB type.

For most part of GRBs both time resolved and time integrated spectra are well described by two-component Band function in the low energy region ${ }^{18}$. Band function first component is proportional to combination of power law with index $\alpha$ and exponential cutoff defined by value $\mathrm{E}_{\text {break }}=\mathrm{E}_{\text {peak }} /(2+\alpha)$ and second one is proportional to power law with index $\beta$. The results of time-resolved GRBs spectroscopy investigation give us dependences of Band function parameter $\beta$ on time for 17 GRB with high energy component registered simultaneously by GBM and LAT were discussed in the Ref. 19. The detailed analysis of behavior of these dependences have shown that for discussed type bursts (GRB090323, GRB090328 and GRB090626) parameter $\beta$ was in the region $-2.3 \div-2.6$ and still approximately constant during 

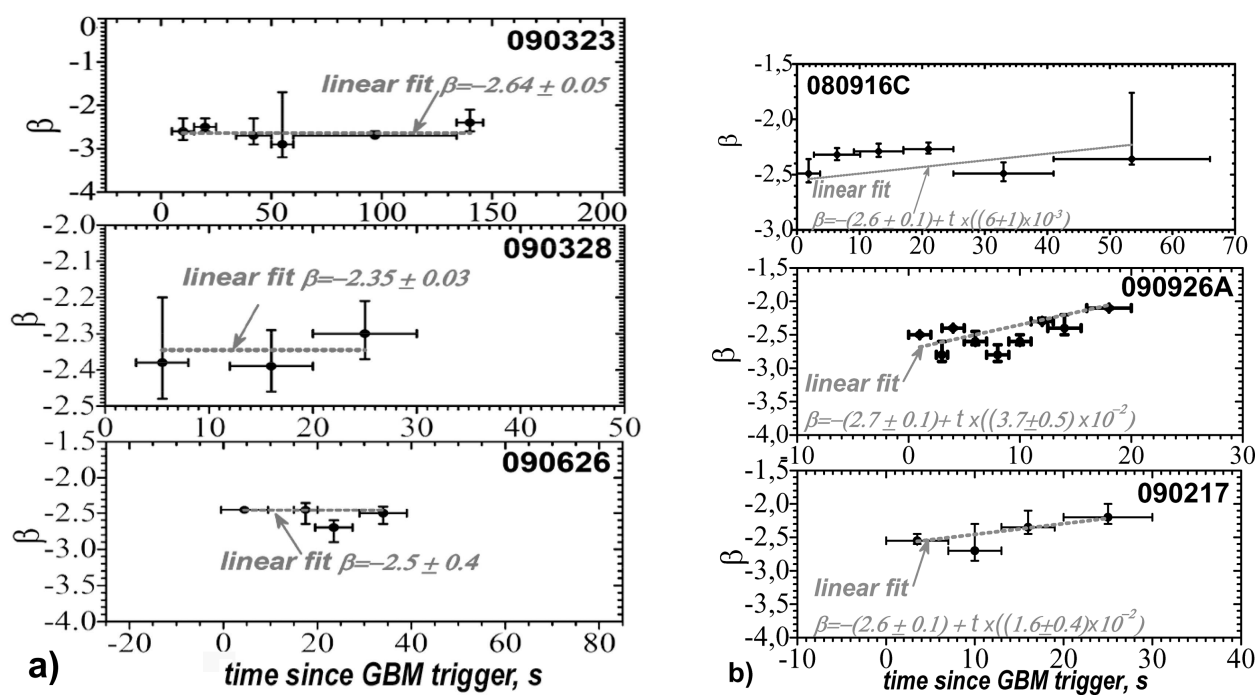

Fig. 3. The evolution of parameter $\beta$ with time for new GRB type (a), for several usual GRB (b) and its linear approximation. Data were taken from Ref. 19.

analyzed event taking into account error boxes — see Fig. 3a. Such behavior is quite different from usual GRB, mostly of which appear significant variations of the parameter $\beta$ with time according to the results of preliminary data analysis - see Fig. 3b. Thereby, the results of detailed spectral analysis give additional evidences to consider GRB090323, GRB090328 and GRB090626 as new subclass of long burst with extended high energy $\gamma$-emission.

\section{Conclusion}

GRB090323, GRB090328 and GRB090626 temporal profiles behavior detailed analysis results have shown that several properties of these events characteristics sufficiently differ from ones of other long GRBs with extended high energy $\gamma$-emission. In contradiction to other bursts, temporal profiles of GRB090323, GRB090328 and GRB090626 have additional maxima after low energy t90 intervals finished. These maxima preceded of faint peaks in low energy bands close to the ends of t90 intervals in low energy bands. Moreover, these events low energy spectral index $\beta$ behavior was quite different from usual GRB according to the results of preliminary data analysis. Thus discussed properties of GRB090323, GRB090328 and GRB090626 temporal profiles and spectrum behavior provide several opportunities to consider these events as new long burst with extended high energy $\gamma$-emission subclass.

\section{References}

1. E. J. Schneid, D. L.ertsch, C. E. Fichtel, et al, Astron. Astroph., 255, no. 1-2, L13 (1991). 
2. B. L. Dingus, J. R. Catelli et al, AIP Conf. Proc., 428, 349 (1998).

3. J. E. McEnery, R.Atkins, W. Benbow et al, AIP Conf. Proc., 526, 240 (2000).

4. I. V. Arkhangelskaja, A. I. Arkhangelsky; A. S. Glyanenko et al Cosmic Research, 45 (issue 3), 261 (2007).

5. I. V. Arkhangelskaja, A. I. Arkhangelsky; A. S. Glyanenko et al, The GRB detected by AVS-F apparatus onboard CORONAS-F satellite in 2001-2005 years, in Proc. of the MG11 Meeting on General Relativity, eds. H. Kleinert, R. T. Jantzen, R/ Ruffini (World Scientific Pub., Singapore, 2008), p. 1968.

6. M. Tavani, G. Barbiellini, A. Argan et al, Astron. Astroph., 502 (issue 3), 995 (2009).

7. Fermi LAT and GBM collab. (J. Granot et al), arXiv:1003.2452 v1 (2010).

8. G. Barbiellini, G. Fedel, F. Lielloet al, Nucl. Instr. and Meth. in Phys.Res. Sect. A, 490 (issue 1-2), 146 (2002)

9. W. B. Atwood, A. A. Abdo, M. Ackermann et al, Astroph. J. , 697 (issue 2), 1071 (2009).

10. E. DelMonte, G. Barbiellini, F. Fuschino et al, Nucl. Instr. and Meth. in Phys.Res. Sect. A, 630 (issue 1), 155 (2011)

11. G. Ghisellini, G. Ghirlanda, L. Nava et al, Mont. Not. of RAS, 403 (issue 2), 926, (2010).

12. M. Marisaldi, G. Barbiellini, E. Costaet al, arXiv:0906.1446 (2009)

13. J. Granot, for the Fermi LAT Collaboration; the GBM Collaboration, arXiv:1003. $2452 \mathrm{v} 1(2010)$

14. I.V. Arkhangelskaja, The properties of GRB with high energy emission, 38th COSPAR Scientific Assembly. Held 18-15 July 2010, in Bremen, Germany, p.2 (2010).

15. V. Vasileiou, F. Piron, D. Tierney et al, GCN circular \#12188, (2011).

16. M. Ackermann, M. Ajello, L. Baldini et al, Astroph. J., 717, L127 (2010)

17. A. Zenin, I. Arkhangelskaja, E. Voevodina et al, The new type of long GRB with high energy gamma emission: GRB090323, GRB090328 and GRB090626?, 32 ICRC, in press (2011).

18. D. Band, J. Matteson, L. Ford et al, Astroph. J., 413, no. 1, 281 (1993).

19. Bin-Bin Zhang, Bing Zhang, En-Wei Liang et al, Astroph. J., 730, 141 (2011). 